\title{
Electrostatic Potential in the tRNA Binding Evolution of Dihydrouridine Synthases
}

\author{
Charles Bou-Nader, ${ }^{\dagger}$ Damien Brégeon, ${ }^{\ddagger}$ Ludovic Pecqueur, ${ }^{\dagger}$ Marc Fontecave, ${ }^{\dagger}$ \\ and Djemel Hamdane, ${ }^{* \dagger}$ \\ ${ }^{\dagger}$ Laboratoire de Chimie des Processus Biologiques, CNRS-UMR 8229, Collège De France, 11 place Marcelin Berthelot, Paris 75231 \\ Cedex 05, France \\ ${ }^{\ddagger}$ CNRS, IBPS, Biology of Aging and Adaptation, Sorbonne Université, 7 quai Saint Bernard, Paris 7525 Cedex 05, France
}

ABSTRACT: Dihydrouridine (D) is an abundant modified base of tRNA found in the majority of living organisms. This base is synthesized via an NADPH-dependent reduction of specific uridines by the dihydrouridine synthases (Dus), a large family of flavoenzymes comprising eight subfamilies. Almost all of these enzymes function with only two conserved domains, an N-terminal catalytic domain (TBD) adopting a TIM barrel fold and a unique C-terminal helical domain (HD) devoted to tRNA recognition, except for the animal U20-specific Dus2 enzyme. Curiously, this enzyme is distinguished from paralogues and its fungi orthologues by the acquisition of an additional domain, a double stranded RNA binding domain (dsRBD), which serves as the main tRNA binding module. On the basis of a homology model of yeast Dus2 and the crystallographic structure of a human Dus2 variant (TBD $+\mathrm{HD}$ ) lacking dsRBD, we herein show

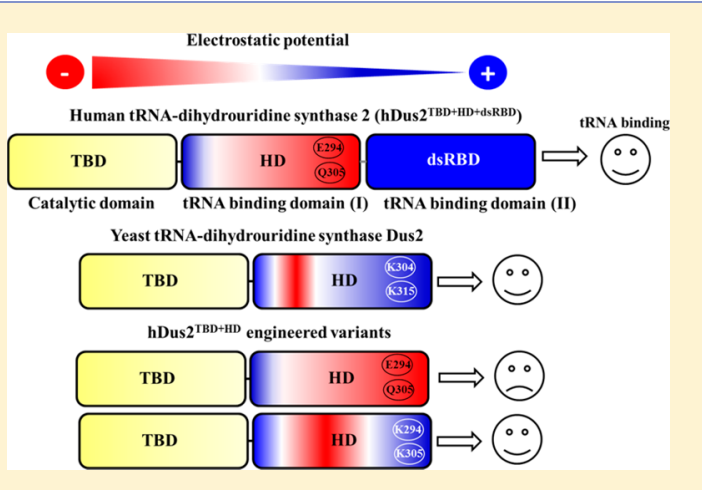
that the HD surface of the human enzyme is less electropositive than that of its yeast orthologue. This is partly due to two positively charged residues, K304 and K315, present in yeast and more broadly in fungi Dus2 that are replaced by E294 and Q305 in human and conserved among animals Dus2. By artificially reintroducing these positive charges in human Dus2 lacking dsRBD, we restored a functional tRNA binding in this enzyme variant. Altogether, these results suggest that the electrostatic potential changes of HD have likely played a key role in the emergence of a new tRNA binding mode among Dus 2 enzymes.

tructural modularity has emerged as an essential adaptive $\checkmark$ feature as a new functional requirement in many enzymes. $^{1-3}$ Typically, enzymatic modularity implies the existence of several domains, whose number increases according to the complexity of organisms. ${ }^{4}$ Although modularity can serve various purposes, ${ }^{3}$ it is often used by enzymes to separate the catalytic function from that of substrate recognition. Nucleic acid-targeting enzymes are known for carrying a particularly complex modularity, whose degree of complexity perfectly correlates with the biological complexity of organisms. Enzymes that mature and functionalize RNAs such as amino-acyl tRNA synthetases, ${ }^{5,6}$ RNases, ${ }^{7,8}$ or the large family of RNA editing and modification enzymes, ${ }^{9}$ to mention only a few of them, obey to this evolution's principle.

Belonging to tRNA-modifying enzymes, dihydrouridine synthases (Dus) catalyze the reduction of the $\mathrm{C} 5=\mathrm{C} 6$ double bond of uridines present at different sites on tRNAs to generate dihydrouridine (D) (Figure 1A,B).

Dihydrouridine is important for stabilizing RNA functional fold, ${ }^{11,12}$ and it has been shown that tRNAs lacking this modified base in combination with others are rapidly degraded by cellular RNases. ${ }^{13}$ Dus form a broad family of modular flavoenzymes that use the flavin mononucleotide (FMN) as a prosthetic group and NADPH coenzyme as a source of hydride. ${ }^{14,15}$ There are eight Dus subfamilies distributed across the living world. ${ }^{15}$ Three are found in bacteria (DusA, B, and C), one in archaea, and four in eukaryotes (Dus1, 2, 3, and 4). So far, only a few of these enzymes have been studied, while most of them remain poorly characterized despite their significant biological relevance. Regarding the function, in vivo and in vitro studies of Escherichia coli, ${ }^{16-18}$ Thermus thermophilus, $^{19,20}$ and Saccharomyces cerevisiae ${ }^{21-23}$ enzymes allowed the substrate specificity of Dus subfamilies to be established (Figure 1B). In addition, bioinformatics analysis ${ }^{15}$ and recent X-ray crystal structures ${ }^{10,18,19,24,25}$ established that the majority of Dus enzymes function only with two domains. These are, namely, an N-terminal domain serving as a catalytic module organized into a TIM-barrel wherein FMN lies in its center (TIM barrel domain = TBD) and a C-terminal domain formed by $\alpha$ helices and used as a tRNA binding module (helical domain $=\mathrm{HD}$ ) (Figure $1 \mathrm{C}$ ). Thus, $\mathrm{TBD}+\mathrm{HD}$ constitutes the canonical Dus structure. Intriguingly, we have recently shown that human dihydrouridine synthase 2 (hDus2) 


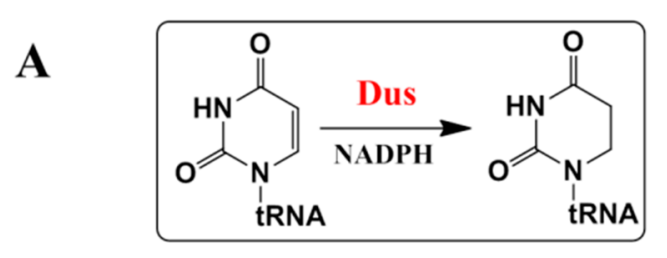

C DusA, B, C, Dus1, Plants and fungi Dus2, Dus4
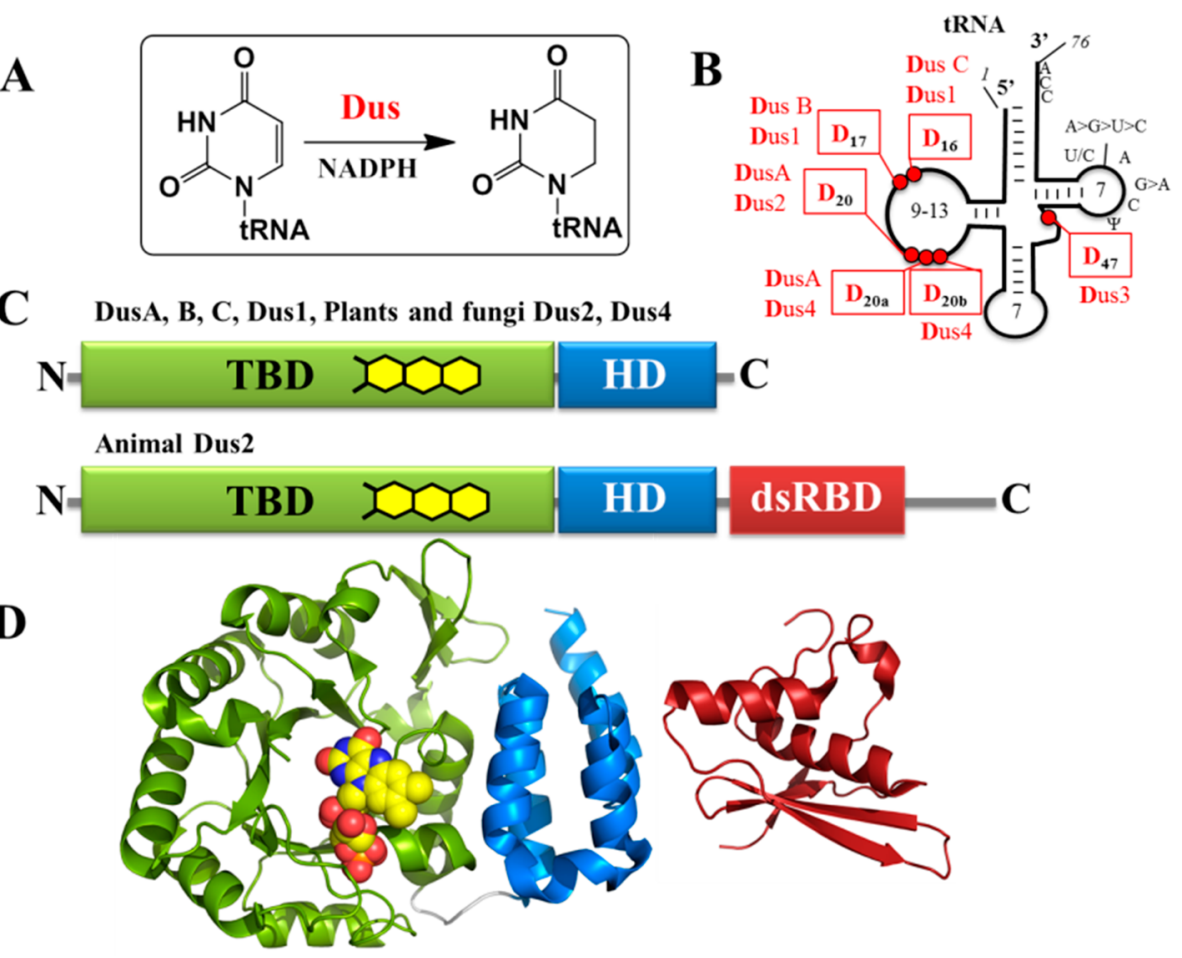

Figure 1. Dihydrouridine synthases and substrate specificities. (A) Reaction catalyzed by Dus enzymes. This reaction consists of a reduction of the $\mathrm{C} 5=\mathrm{C} 6$ uridine double bond by $\mathrm{FMNH}^{-}$(flavin hydroquinone), which results from the preliminary reduction of FMN by NADPH. (B) Substrate specificities of Dus. Secondary structure of tRNA showing the uridine targeted (red circles) by Dus enzymes to form D (red boxed). DusA, B, and C are bacterial enzymes. DusA catalyzes the formation of D20 and D20a, while Dus B and C form D17 and D16, respectively. Dus1, 2, 3, and 4 are eukaryotic enzymes. Dus1 and Dus4 are bisite-specific enzymes synthesizing D16/D17 and D20a/D20b, respectively, while Dus2 and Dus3 synthesize D20 and D47, respectively. (C) Domain modularity of Dus enzymes. All Dus enzymes contain an N-terminal catalytic domain organized into a TIM Barrel (TBD, green), which binds FMN coenzyme (yellow), and a helical domain (HD, blue) known as the bona fide tRNA binding module. Animal Dus2 carries an additional domain placed after the HD, the dsRBD (red), which functions as the main tRNA binding module. ${ }^{10}$ Note that Dus3 is a particular case among Dus since, although it carries TBD + HD, this enzyme has nonetheless a nonconventional size with more than 700 residues. (D) Crystallographic X-ray structure of hDus2. The left panel shows the canonical Dus structure (TBD + HD named as hDus2 ${ }^{\mathrm{TBD}+\mathrm{HD}}$, PDB 4WFS) while the right panel shows the dsRBD (hDus2 ${ }^{\mathrm{dsRBD}}$, PDB 4WFT). The domains are colored according to panel C.

and, based on sequence alignments, probably all animal Dus2 display a more complex modularity than its Dus paralogues or orthologues. ${ }^{10,15}$ In addition to the canonical Dus structure, we showed that this enzyme possesses a third module, the double stranded binding domain (dsRBD), appended at the Cterminus of $\mathrm{HD}$, and an additional $\sim 50$ amino acid peptide predicted as intrinsically disordered that ends the protein (Figure 1C,D). ${ }^{10}$ Moreover, this dsRBD seems to have evolved to function as the major tRNA binding domain of hDus2, suggesting that during evolution the tRNA binding function has been transferred, at least in part, from the TBD + HD part to the dsRBD. Why animal Dus2 specifically enjoys such a complex modularity while all other Dus and notably their closest relative fungi Dus2 function without dsRBD remains a mystery. It is more puzzling that this dsRBD does not seem to ensure a better thermodynamic stability of the tRNA/Dus2 complex since hDus2 and its yeast orthologue Dus2p (Dus2 from Saccharomyces cerevisiae) share a similar affinity for tRNA. ${ }^{10}$ However, one cannot exclude that this modularity could serve other cellular purposes, which remain to be demonstrated. Indeed, in addition to its tRNA modification function, human Dus2 has been shown to promote cell growth in some cancers mediated via its ability to interact with the amino-acyl tRNA synthetase complex EPRS or protein kinase R. However, its exact pro-oncotic mechanism is not yet elucidated. $^{26,27}$
Using a comparative approach combining bioinformatics, structural and functional analysis, we here identified that $\mathrm{HD}$ of bimodular Dus2, specific to fungi, presents a more electropositive potential than that of its trimodular Dus2 orthologue, specific to animals. By reintroducing these lacking positive charges into the $\mathrm{HD}$ of a truncated hDus2 version (hDus $2^{\mathrm{TBD}+\mathrm{HD}}$ ) in which both dsRBD and C-terminal region were removed, we managed to generate a new functional canonical Dus2 carrying a better affinity for tRNA than its parent counterpart. These results lead us to speculate that this important physicochemical electrostatic property has played a major role in the evolutionary process that led to the complex modularity of current animal Dus2.

\section{MATERIALS AND METHODS}

Protein Purification. Wild-type human Dus2 (hDus2) and $\Delta(2-13)$-hDus $2^{\mathrm{TBD}+\mathrm{HD}}$, a construct lacking residue Ile2-Lys13 and Q341-W493, were cloned in pET11d vector between BamHI and NcoI while hDus2 $2^{\mathrm{TBD}+\mathrm{HD}}$, a construct lacking Q341-W493, and Dus2p (Dus2 from Saccharomyces cerevisiae) were cloned in pET15b between BamHI and NcoI. All constructs were expressed in BL21(DE3)pLysS (Novagen) in LB medium. Induction with $0.5 \mathrm{mM}$ isopropyl-1-thio- $\beta$-Dgalactopyranoside (IPTG) was done at $\mathrm{OD}_{600} \sim 0.6$ and left overnight at $29^{\circ} \mathrm{C}$. Pelleted cells were suspended in buffer A (50 mM sodium phosphate $\mathrm{pH} 8,2 \mathrm{M} \mathrm{NaCl}, 25 \mathrm{mM}$ 
imidazole, and $10 \%$ glycerol) with the addition of phenylmethane sulfonyl fluoride (PMSF) and lysed by sonication followed by centrifugation for $40 \mathrm{~min}$ at $\sim 148000 \mathrm{~g}$. The supernatant was loaded on a NiNTA column (GE healthcare) washed several times with buffer A and eluted with buffer A supplemented with $250 \mathrm{mM}$ imidazole. All proteins were further purified by a size exclusion chromatography step on a Superdex HiLoad S200 16-600 unit (GE healthcare). Purity was assessed by SDS-PAGE. Point mutations were carried out with the Q5 site-directed mutagenesis kit (New England BioLabs) following the recommended procedure.

Preparation of Bulk tRNA. To extract bulk tRNA from yeast strain BY4741 $\Delta$ dus2 derivative (MATa; his $3 \Delta 1$; leu2 $\Delta$ 0 ; met $15 \Delta 0$; ura3 $\Delta 0$; YNR015w::kanMX4), cells were grown in $500 \mathrm{~mL}$ of YPD (peptone $2 \%$; yeast extract $1 \%$ and glucose $2 \%)$ to an $\mathrm{OD}_{600}$ of 0.8 . Pelleted cells were washed twice in 20 $\mathrm{mL}$ of TMN (10 mM Tris- $\mathrm{HCl}\left(\mathrm{pH} \mathrm{7.5),} 10 \mathrm{mM} \mathrm{MgCl}_{2}\right.$, $0.15 \mathrm{M} \mathrm{NaCl}$ ). The final pellet was resuspended in $20 \mathrm{~mL}$ of TMN and $20 \mathrm{~mL}$ of acidic phenol ( $\mathrm{pH} \mathrm{4.5)}$ and incubated for $20 \mathrm{~min}$ at room temperature on a rotating wheel. After centrifugation, the aqueous phase was recovered, supplemented with $\mathrm{LiCl}$ to a final concentration of $0.8 \mathrm{M}$, and incubated overnight at $4{ }^{\circ} \mathrm{C}$ to precipitate high molecular mass molecules. The precipitate was eliminated by centrifugation, and the supernatant was supplemented with two volumes of $100 \%$ ethanol and incubated at $-20{ }^{\circ} \mathrm{C}$ for $2 \mathrm{~h}$ to precipitate tRNAs. After centrifugation, pelleted tRNAs were washed twice in $70 \%$ ethanol and resuspended in $1 \mathrm{~mL}$ of RNase-free water. tRNAs were desalted and concentrated four times to 50 $\mu \mathrm{L}$ in Centricon YM-3 devices (Millipore) using $100 \mathrm{mM}$ ammonium acetate (pH 5.3) as a final buffer.

Crystallization, Data Collection, Structure Determination, and Refinement. Crystals of $\Delta(2-13)$ hDus $2^{\mathrm{TBD}+\mathrm{HD}}$ E294K mutant were obtained by vapor diffusion by mixing $1 \mu \mathrm{L}$ of freshly prepared protein in $50 \mathrm{mM}$ Tris $\mathrm{pH}$ 8 and $150 \mathrm{mM} \mathrm{NaCl}$ with $1 \mu \mathrm{L}$ of reservoir comprised of $30 \%$ PEG 2000 MME, $200 \mathrm{mM}$ ammonium sulfate, and $50 \mathrm{mM}$ sodium acetate $\mathrm{pH}$ 5.5. Crystallization of $\Delta(2-13)$ hDus $2^{\mathrm{TBD}+\mathrm{HD}}$ Q305K mutant required $2.2 \mathrm{M}$ ammonium sulfate, while $\Delta(2-13)$-hDus2 $2^{\mathrm{TBD}+\mathrm{HD}}$ E294K/Q305K mutant crystallized in 30\% PEG $2000 \mathrm{MME}, 200 \mathrm{mM}$ ammonium sulfate, and $50 \mathrm{mM}$ sodium acetate $\mathrm{pH}$ 5. Crystals were obtained after 2 days and were cryoprotected using 15\% glycerol before flash freezing in liquid nitrogen. The three structures were phased by molecular replacement using Phaser $^{28}$ and as a starting model PDB entry 4XP7. All diffraction data were collected at $100 \mathrm{~K}$ on single crystals at the microfocused beamline PROXIMA-2 equipped with an Eiger X-9 M detector at SOLEIL synchrotron (Saint-Aubin, France). Data were indexed using XDS, scaled and merged with STARANISO using the aP_scale module of autoPROC to correct the anisotropy of the data. ${ }^{29}$ The three structures were refined with autoBUSTER, ${ }^{30}$ and manual building into the density was performed in Coot. ${ }^{31}$ TLS were used with one TLS group per protein chain. STARANISO corrected intensities and structure factors as well as uncorrected intensities and structure factors without resolution cutoff have been deposited in the Protein Data Bank along with the coordinates and Fourier coefficients. (See Table S1 for the determination of best-fit ellipsoid to the anisotropic cutoff surface.)

Homology Modeling and Electrostatic Potential Analysis. Homology models of Dus2p were built by homology-modeling servers, I-TASSER, ${ }^{32}$ SWISS-MODEL, ${ }^{33}$ Phyre2, ${ }^{34}$ and ROBETTA, ${ }^{35}$ that all used PDB 4XP7 as a template. The resulting models were validated by the server ProQ3D. ${ }^{36}$ The calculation of protein electrostatic surfaces was performed by software APBS (v1.4). ${ }^{37}$ Before calculations, missing atoms from the side chains were modeled using the PDB2PQR software. ${ }^{38}$ Calculations were performed at $310 \mathrm{~K}$ with $150 \mathrm{mM} \mathrm{NaCl}$ with the same grid size $(193,193,161)$ for all calculations. For all of the representations, we choose to show an electrostatic potential within $\pm 3 \mathrm{kTe}^{-1}$.

tRNA Binding Assay. Electrophoretic mobility shift assays were carried out on a $6 \%$ native (19:1) PAGE at $4{ }^{\circ} \mathrm{C}$ with 100 $\mathrm{V}$. Increased concentrations of proteins were added to a fix concentration of bulk tRNA lacking D20 ( $1 \mu \mu \mathrm{M})$ and incubated at room temperature for $20 \mathrm{~min}$ in $50 \mathrm{mM}$ Tris $\mathrm{pH} 8,10 \%$ glycerol, $5 \mathrm{mM}$ DTT, and $150 \mathrm{mM}$ ammonium acetate prior to migration. RNA was visualized by toluidine coloration and quantified using ImageJ (https://imagej.nih. gov/ij/index.html).

NADPH Oxidase Activity. Oxidase activities were assessed under steady state conditions with menadione as a final electron acceptor in $50 \mathrm{mM}$ sodium phosphate $\mathrm{pH} 8,150 \mathrm{mM}$ $\mathrm{NaCl}$, and $20 \%$ glycerol at $23{ }^{\circ} \mathrm{C}$. Two $\mu \mathrm{M}$ of holoprotein was mixed with various concentrations of NADPH and $200 \mu \mathrm{M}$ menadione. NADPH oxidation was followed by the decrease in absorption at $340 \mathrm{~nm}$ using a Cary-50 spectrophotometer. Data were analyzed using Michaelis-Menten formalism.

Dihydrouridine Activity Assay. In vitro activity was assayed for $30 \mathrm{~min}$ at $30{ }^{\circ} \mathrm{C}$ in $50 \mathrm{mM}$ Tris $\mathrm{pH} 8,150 \mathrm{mM}$ ammonium acetate, $1 \mathrm{mM}$ dithiothreitol, $2 \mathrm{mM} \mathrm{MgCl} 2,10 \%$ v/v glycerol. Bulk tRNAs $(100 \mu \mathrm{M})$ were incubated with $5 \mu \mathrm{M}$ of protein in a total volume of $50 \mu \mathrm{L}$, and the reaction was started with the addition of $2 \mathrm{mM} \mathrm{NADPH}$. Quenching was performed by adding $50 \mu \mathrm{L}$ of acidic phenol (Sigma-Aldrich) followed by centrifugation at $\sim 12000 \mathrm{~g}$ for $10 \mathrm{~min}$, and tRNA in the aqueous phase was ethanol precipitated and further purified using a MicroSpin G-25 column (GE-healthcare). Dihydrouridine quantification was carried out by means of a colorimetric method as described previously. ${ }^{10}$ Briefly, samples were incubated at $40{ }^{\circ} \mathrm{C}$ for $30 \mathrm{~min}$ after the addition of $5 \mu \mathrm{L}$ of $1 \mathrm{M} \mathrm{KOH}$. The solutions were neutralized with $25 \mu \mathrm{L}$ of $96 \% \mathrm{H}_{2} \mathrm{SO}_{4}$ followed by $25 \mu \mathrm{L}$ of a $3 \%$ solution of $2,3-$ butanedione monoxime (Sigma-Aldrich) and $25 \mu \mathrm{L}$ of a saturated solution of N-Phenyl-p-phenylenediamine (SigmaAldrich). Samples were then heated at $95{ }^{\circ} \mathrm{C}$ for $10 \mathrm{~min}$ and cooled to $55{ }^{\circ} \mathrm{C}$. Following the addition of $50 \mu \mathrm{L}$ of $1 \mathrm{mM}$ $\mathrm{FeCl}_{3}$, a violet-red coloration appeared allowing quantification via absorption at $550 \mathrm{~nm}$. D content in tRNA was determined by using a standard curve obtained with variable amounts of dihydrouracil.

\section{RESULTS}

Human Dus2 Carries a More Electronegative HD than Its Yeast Orthologue. All nucleic acid enzymes acting on tRNA recognize their substrates primarily via positively charged residues such as arginine and lysine. This led us to speculate that the low affinity of hDus $2^{\mathrm{TBD}+\mathrm{HD}}$ for tRNA may result from the absence of one or more positively charged residue at the TBD or HD surface or both. One way to address this issue is to compare the electrostatic surfaces of both hDus $2^{\mathrm{TBD}+\mathrm{HD}}$ and Dus2 $\mathrm{p}$ and identify regions that present different charge distributions. In the case of hDus $2^{\mathrm{TBD}+\mathrm{HD}}$, we used the crystal structure of $\Delta(2-13)-\mathrm{hDus} 2^{\mathrm{TBD}+\mathrm{HD}}$ as a 
Table 1. NADPH Oxidase Activity of hDus2 $2^{\mathrm{TBD}+\mathrm{HD}}$ and Its Mutants Using Menadione As a Final Electron Acceptor

\begin{tabular}{lccc}
\multicolumn{1}{c}{ protein } & $k_{\text {cat }}\left(\mathrm{s}^{-1}\right)$ & $K_{\mathrm{M}}(\mu \mathrm{M})$ & $\begin{array}{c}k_{\text {cat }} / K_{\mathrm{M}} \\
\left(\mu \mathrm{M}^{-1} \mathrm{~s}^{-1}\right)\end{array}$ \\
hDus2 $^{\mathrm{TBD}+\mathrm{HD}}$ & $0.8 \pm 0.05$ & $63 \pm 5$ & 0.013 \\
E294K hDus2 $2^{\mathrm{TBD}+\mathrm{HD}}$ & $0.7 \pm 0.08$ & $70 \pm 10$ & 0.01 \\
Q305K hDus2 $^{\mathrm{TBD}+\mathrm{HD}}$ & $0.7 \pm 0.04$ & $68 \pm 6$ & 0.01 \\
E294K/Q305KhDus2 & $1 \pm 0.06$ & $55 \pm 5$ & 0.018 \\
\hline
\end{tabular}

template, a version wherein $12 \mathrm{~N}$-terminal residues were removed to promote crystallization, whereas for Dus $2 p$ there is so far no structure available to conduct such an analysis. To overcome this issue, we generated homology models of Dus $2 p$ from four different homology-modeling servers I-TASSER, SWISS-MODEL, Phyre2, and ROBETTA that all used human Dus2 as a template, the yeast enzyme having $~ 37 \%$ sequence identity with its orthologue hDus2 ${ }^{\mathrm{TBD}+\mathrm{HD}}$ (Figure S1A). We choose the best models from each method and then validated their accuracy with ProQ3D. The local accuracy of each model is visualized directly on their $3 \mathrm{D}$ representation (Figure S1B). As shown in Figure S1B, all of the models present high validation scores for the TBD and HD domains except for some areas that correspond to loops and linker, namely, (i) the $\beta 4-\beta 5$ loop (V61-T71); (ii) the active site loop (G116-A130), which is known to be flexible and not visible in both available $\mathrm{X}$-ray structures of hDus2 $2^{\mathrm{TBD}+\mathrm{HD}}$ (PDBs: 4WFS and 4XP7); (iii) the linker connecting TBD to HD (T261-S271) (Figure S2). The good quality of these models allowed us to perform a comparative structural analysis with $\Delta(2-13)-\mathrm{hDus} 2^{\mathrm{TBD}+\mathrm{HD}}$. As expected, the $\mathrm{N}$-terminal region is organized around a $\alpha 8$ / $\beta 11$ TIM barrel fold wherein the central barrel formed by eight parallel $\beta$ strands is surrounded by $8 \alpha$ helices. Like in the human enzyme, the TIM barrel core diverges from the classical $\alpha 8 / \beta 8$ fold due to a structural insertion organized into an antiparallel $\beta$ sheet $(\beta 3-\beta 5)$ whose primary sequence appears similar in both Dus2p and hDus2 (Figure S1A). Interestingly, this antiparallel $\beta$ sheet is not observed in paralogous and orthologous bacterial Dus, suggesting that this structural element could be a specific feature of the Dus2 family. Finally, we can notice that the length of the $\beta 4-\beta 5$ loop of Dus $2 \mathrm{p}$ is six residues longer than that of hDus 2 and that its size seems to be variable among fungi Dus2 (Figure S4A).

Comparison of the electrostatic surface of $\Delta(2-13)$ hDus $2^{\mathrm{TBD}+\mathrm{HD}}$ and Dus $2 \mathrm{p}$ models showed that TBDs share a rather similar charge distribution (Figure S3), although slight differences can be observed in the $\beta 4-\beta 5$ loop, active site loop, and TBD-HD linker due to the weak confidence of the models for these regions (Figure S2). However, HDs appear to be clearly different. In particular, one of hDus2 is much less electropositive than that of its yeast counterpart. This difference is explained in part by the fact that two lysines, $\mathrm{K} 304$ and $\mathrm{K} 315$, present in yeast $\mathrm{HD}$ are lost in the human enzyme and replaced by the negatively charged E294 and polar Q305, respectively. Dus2 sequence analysis revealed that K304 and K315 are conserved among fungi (Figure S4A), while E294 and Q305 are conserved only among animals (Figure S4B).

We previously showed that electrostatic surface of hDus2 $\mathrm{HD}$ is less electropositive than its bacterial orthologue DusA and paralogue DusC. Interestingly, here, the analysis of all bacterial Dus revealed that two positive charges located at the

Table 2. Summary of Data Collection and Refinement Statistics ${ }^{a}$

\begin{tabular}{|c|c|c|c|}
\hline & HsDus $2^{\mathrm{TBD}+\mathrm{HD}}$ & HsDus2 $2^{\mathrm{TBD}+\mathrm{HD}}$ & HsDus $2^{\mathrm{TBD}+\mathrm{HD}}$ \\
\hline & E294K mutant & Q305K mutant & E294K Q305K double mutant \\
\hline & PDB 6EZA & PDB 6EZB & PDB 6EZC \\
\hline \multicolumn{4}{|c|}{ Data Collection } \\
\hline space group & $P 2(1)$ & I 222 & $P 2(1) 2(1) 2(1)$ \\
\hline cell dimensions $a, b, c(\AA)$ & $55.47,77.11,84.48$ & $72.08,84.11,144.35$ & $45.99,84.66,100.39$ \\
\hline cell dimensions $\alpha, \beta, \gamma(\mathrm{deg})$ & $90,91.99,90$ & $90,90,90$ & $90,90,90$ \\
\hline resolution $(\AA)$ & $45.00-2.00(2.097-2.00)$ & $43.61-2.25(2.452-2.250)$ & $43.18-2.00(2.11-2.00)$ \\
\hline$R_{\text {sym }}$ or $R_{\text {merge }}$ & $0.142(1.179)$ & $0.137(1.609)$ & $0.161(2.521)$ \\
\hline$R_{\text {meas }}$ & $0.160(1.344)$ & $0.145(1.714)$ & $0.170(2.665)$ \\
\hline$I / \sigma I$ & $8.0(1.3)$ & $12.6(1.4)$ & $10.1(1.1)$ \\
\hline completeness $(\%)^{*}$, spherical & $78.5(29.3)$ & $78.1(18.6)$ & $82.9(27.0)$ \\
\hline completeness $(\%)^{*}$, ellipsoidal & $93.1(93.2)$ & $93.4(60.8)$ & $89.9(43.5)$ \\
\hline redundancy & $4.7(4.3)$ & $9.2(8.5)$ & $9.3(8.9)$ \\
\hline $\mathrm{CC}(1 / 2)$ & $0.994(0.517)$ & $0.998(0.385)$ & $0.997(0.413)$ \\
\hline \multicolumn{4}{|c|}{ Refinement } \\
\hline resolution $(\AA)$ & $38.55-2.00$ & $43.61-2.25$ & $42.33-2.00$ \\
\hline no. of reflections & 37935 (759) & $16301(418)$ & $22658(454)$ \\
\hline$R_{\text {work }} / R_{\text {free }}$ & $0.191 / 0.227$ & $0.181 / 0.223$ & $0.182 / 0.223$ \\
\hline no. of atoms, protein & 4805 & 2344 & 2368 \\
\hline no. of atoms, ligand/ion & 116 & 57 & 79 \\
\hline no. of atoms, water & 359 & 41 & 230 \\
\hline$B$-factor, macromolecule & 32.93 & 55.67 & 38.56 \\
\hline$B$-factor, ligand/ion & 33.11 & 49.70 & 43.33 \\
\hline$B$-factor, water & 38.62 & 48.02 & 47.67 \\
\hline rms deviation, bond lengths $(\AA)$ & 0.010 & 0.010 & 0.010 \\
\hline rms deviation, bond angles ( $\mathrm{deg}$ ) & 1.06 & 1.13 & 1.03 \\
\hline
\end{tabular}

${ }^{a}$ Each data set was collected from a single crystal. Data statistics are calculated with STARANISO. 

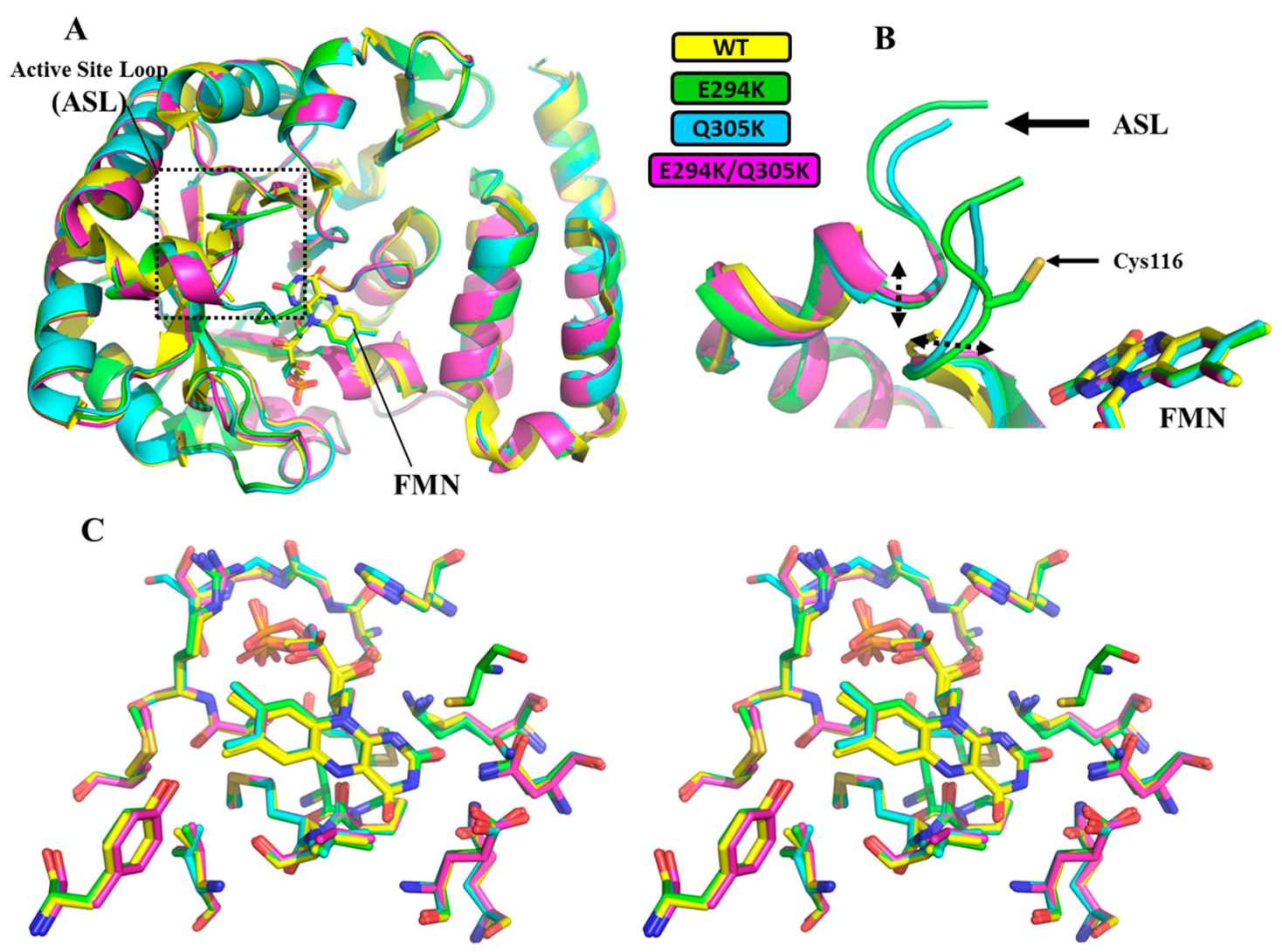

Figure 2. Structural alignment of the $\mathrm{X}$-ray structures of $\Delta(2-13)-\mathrm{hDus} 2^{\mathrm{TBD}+\mathrm{HD}}$ and its $\mathrm{HD}$ mutants. (A) Structural alignment of the overall structure of $\Delta(2-13)$-hDus2 ${ }^{\mathrm{TBD}+\mathrm{HD}}$ carrying a wild-type HD (yellow, PDB: 4WFS), E294K hDus2 $2^{\mathrm{TBD}+\mathrm{HD}}$ (green), Q304K hDus2 ${ }^{\mathrm{TBD}+\mathrm{HD}}$ (blue), and E294K/Q304K hDus2 ${ }^{\mathrm{TBD}+\mathrm{HD}}$ (magenta). FMN, lying at the center of the TBD, is shown as sticks. RMSD for E294K, Q305K, and E294K/ Q305K mutants versus 4WFS are $0.22 \AA$ over $279 \mathrm{CA}$ atoms, $0.27 \AA$ over $282 \mathrm{CA}$ atoms, and $0.25 \AA$ versus $272 \mathrm{CA}$ atoms, respectively. (B) Zoom on the active site loop (ASL) containing the catalytic Cys116 playing the role of acid in the Dus-catalyzed reaction. Only E294K hDus2 $2^{\mathrm{TBD}+\mathrm{HD}}$ shows a completely visible Cys116. (C) Stereo view of the structural alignment between wild-type (yellow, PDB: 4WFS), E294K (green), Q304K (blue), and E294K/Q304K (magenta) hDus2 $2^{\mathrm{TBD}+\mathrm{HD}}$ active sites. The residues represented as sticks are within $4 \AA$ from FMN.
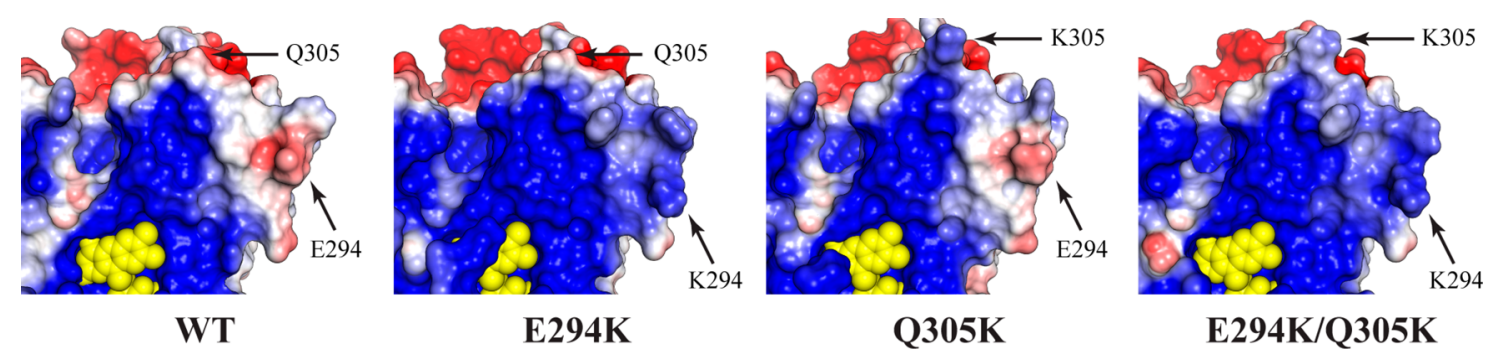

Figure 3. HD Electrostatic surfaces of $\Delta(2-13)$-hDus $2^{\mathrm{TBD}+\mathrm{HD}}$ (PDB: $4 \mathrm{WFS}$ ) and mutants. The electrostatic potentials are calculated with APBS and within $\pm 3 \mathrm{kTe}^{-1}$. The FMN is shown as yellow spheres.

same place as those of Dus2 $p$ are also found in the HD of DusA but absent in both DusB and C (Figure S5). We propose that these two residues likely constitute a signature of the U20specific Dus enzymes in both bacteria and fungi. Taken together, these results suggest that in the family of Dus2 enzymes specifically, the HD has evolved from an electropositive domain in its closest relatives found in fungi to a more electronegative domain in animals, thereby leading to a domain displaying less tRNA binding capacity and loosening the overall binding of tRNA in hDus2 $2^{\mathrm{TBD}+\mathrm{HD}}$.

Flavin Spectrum and NADPH-Oxidase Activity of E294K, Q305K, and E294K/Q305K hDus2 ${ }^{\text {TBD+HD }}$ Mutants. To validate the evolutionary relevance and importance of these residues in the tRNA binding function and to obtain some clues on how the RNA binding function transfer from HD to dsRBD occurred, we proceeded by engineering three hDus2 $2^{\mathrm{TBD}+\mathrm{HD}}$ variants, namely, E294K, Q305K, and E294K/ Q305K. Although residues 294 and 305 appear to be located far from the flavin binding site (Figure S5), we nonetheless ensured that their replacement did not affect the redox reactivity of FMN by first recording the UV-visible spectrum of each mutant and then assessing its NADPH oxidase activity under steady state conditions. As shown in Figure S6, wild-type and variant proteins display a similar flavin spectrum indicating that these mutations do not disturb the electronic environment of FMN. In addition, they catalyze menadione-dependent $\mathrm{NADPH}$ oxidation with apparent $k_{\text {cat }}$ and $K_{\mathrm{M}}$ values at $23{ }^{\circ} \mathrm{C}$, comparable to those for the parent protein (Table 1). 

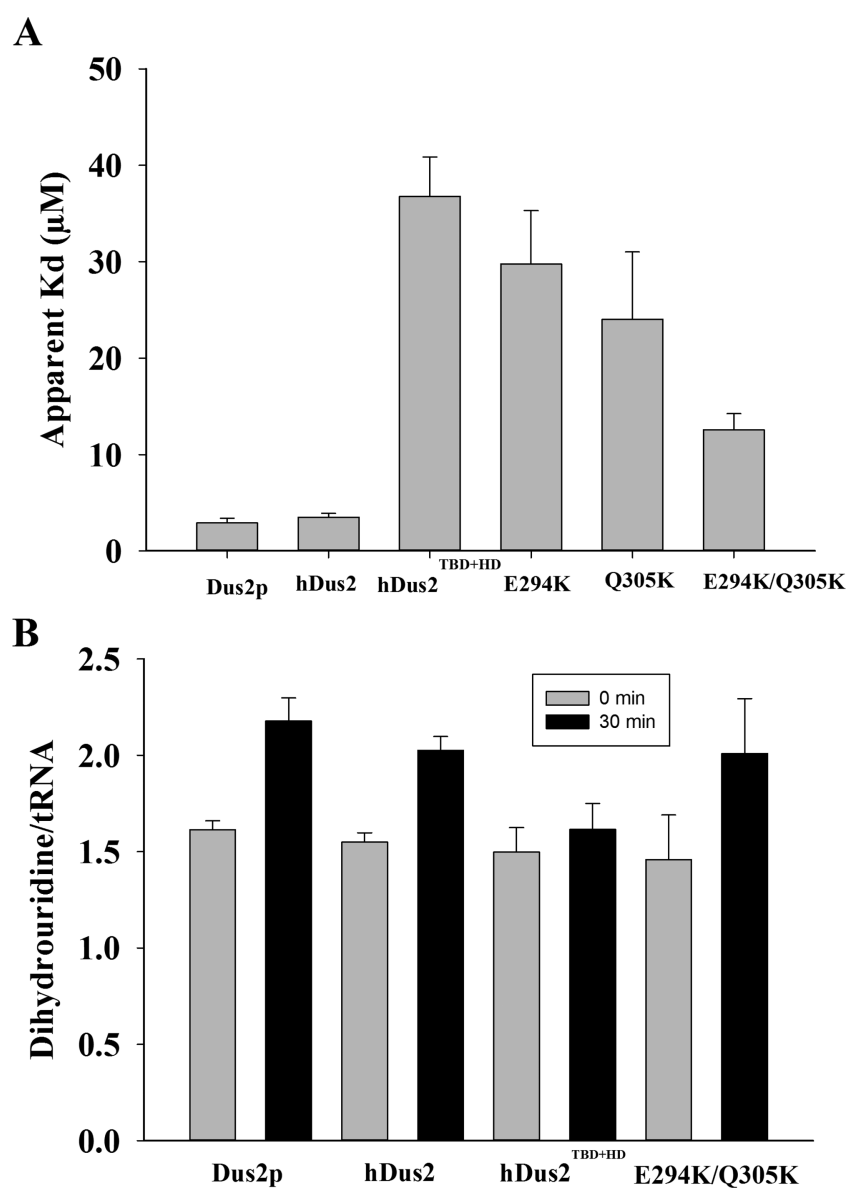

Figure 4. tRNA binding and dihydrouridine synthase activity. (A) Histogram showing $K_{\mathrm{d}}$ values in $\mu \mathrm{M}$ for tRNA of Dus $2 \mathrm{p}$, full-length hDus2 noted as hDus2, hDus2 $2^{\mathrm{TBD}+\mathrm{HD}}$, and its mutants as determined by electrophoretic mobility shift assays. (B) Histogram reporting the amount of D/tRNA in the absence of enzyme or in the presence of Dus + NADPH incubated for 0 or $30 \mathrm{~min}$. The error bars are calculated from three different sets of experiments.

Therefore, E294K, Q305K, and E294K/Q305K mutations do not disturb the FMN or NADPH binding properties of the proteins.

X-ray Structure of E294K, Q305K, and E294K/Q305K hDus2 $2^{\mathrm{TBD}+\mathrm{HD}}$ Mutants. Next, we structurally characterized the mutants by solving their X-ray structures. Since hDus2 $2^{\mathrm{TBD}+\mathrm{HD}}$ did not crystallize, we used $\Delta(2-13)$ hDus $2^{\mathrm{TBD}+\mathrm{HD}}$ to analyze the mutations. E294K, Q305K, and E294K/Q305K $\Delta(2-13)$-hDus2 ${ }^{\mathrm{TBD}+\mathrm{HD}}$ mutants crystallized in $P 2$ (1), I 22 2, and $P$ 2(1) 2(1) 2(1) space groups at 2, 2.25, and $2 \AA$ resolution, respectively (Table 2$)$.

The asymmetric unit of $\mathrm{E} 294 \mathrm{~K}$ protein is formed by two molecules, while Q305K and E294K/Q305K mutants contain only one molecule. Gel filtration analysis showed that like $\Delta(2-13)$-hDus $2^{\mathrm{TBD}+\mathrm{HD}}$, all of the protein mutants are mainly monomers in solution (Figure S7), suggesting that the dimeric asymmetric unit of the E294K mutant is a result of crystal packing. All of the mutants display a structure identical to that of $\Delta(2-13)$-hDus $2^{\mathrm{TBD}+\mathrm{HD}}$. Structural overlay shows no significant conformational changes induced by the mutations (Figure 2A).

Nonetheless, while the strictly conserved cys116 of the active site loop (from 115 to 129), which acts as an acid residue during dihydrouridine synthesis, ${ }^{19,22}$ is too flexible to be observed in our $\Delta(2-13)$-hDus $2^{\mathrm{TBD}+\mathrm{HD}}$ crystal structure, its electronic density is clearly visible in the E294K mutant structure, allowing its manual building (Figure 2B). Lying in the TBD center, FMN is stabilized by several interactions that are all preserved in the mutants (Figure 2C). We then calculated the electrostatic potential of the surfaces of the four hDus2 protein variants. From the electrostatic surface representations, we can clearly observe that these mutations introduce positive charge(s) into $\mathrm{HD}$ (Figure 3), the effect being more prominent for the E294K/Q305K double mutant. Hence, mutations convert the HD into a more electropositive domain without altering the overall structure of $\mathrm{hDus} 2^{\mathrm{TBD}+\mathrm{HD}}$.

E294K, Q305K, and E294K/Q305K hDus2 ${ }^{\mathrm{TBD}+\mathrm{HD}}$ Mutants Increase the tRNA Binding Affinity and Dihydrouridine Activity. To determine if such mutations enhance the tRNA binding function, we performed electrophoretic mobility shift assays on Dus2p from yeast, full-length hDus2, hDus2 $2^{\mathrm{TBD}+\mathrm{HD}}$, and its mutants (Figure S8). Consistent with our previous report, Dus2p and hDus2 seem to share a similar affinity for tRNA (Figures 4A and S9). The hDus $2^{\mathrm{TBD}+\mathrm{HD}}$ has a $\sim 10$-fold lower affinity than hDus2 and Dus2p (Figure 4A). Interestingly, introducing a positive charge at position 294 increases the tRNA affinity of hDus $2^{\mathrm{TBD}+\mathrm{HD}}$ by $\sim 1.24$-fold, while when introduced at position 305 we observed a $\sim 1$.4-fold gain of tRNA binding capacity, which seems to be not a significant improvement. However, the remarkable effect is observed for the double mutant, which exhibits a $\sim 3.6$-fold higher affinity than the parent protein. This result suggests that both charges introduced at positions 294 and 305 act synergistically in tRNA binding. As evidenced from the activity performed in vitro, the gain of the tRNA binding affinity translates into a better dihydrouridine synthase activity, which is comparable to that of full-length hDus2 (Figure 4B).

\section{DISCUSSION}

We have here unambiguously established that the HD domain of hDus2 and more broadly of animals Dus2 are less electropositive than that of its yeast orthologue operating without dsRBD (Figure S1). This deficiency of positive charges explains in part why the canonical hDus $2^{\mathrm{TBD}+\mathrm{HD}}$ structure is no longer able to ensure a stable enzyme/tRNA complex without dsRBD. By engineering an artificially electropositive HD via introduction of the missing positive charges at positions 294 and 305 , conserved in fungi but absent in animals, in hDus $2^{\mathrm{TBD}+\mathrm{HD}}$, we were able to increase its affinity for tRNA. This was associated with an improvement of the dihydrouridine synthase activity, suggesting that these positive charges promote the formation of a catalytically competent enzyme/ tRNA complex. Although the affinity was improved by $~ 3.6-$ fold with the E294K/Q305K double mutant, we did not restore an affinity comparable to that observed with Dus2p (Figure 4A) or full-length hDus2. ${ }^{10}$ This suggests that other residues are certainly contributing to this difference, but in the absence of the Dus2p/tRNA complex structure, their identification remains challenging. Regarding hDus2, structural details on the full-length and tRNA-bound enzyme are also lacking. Nevertheless, based on a number of consistent evidence that includes (i) the structure of $\Delta(2-13)$ hDus $2^{\mathrm{TBD}+\mathrm{HD}}$ and hDus2 $2^{\mathrm{dsRBD}}$, (ii) the general mode of action of dsRBD, which recognizes $\sim 12-16$ bp dsRNA structure, and (iii) the structure of its bacterial orthologue DusA in complex with tRNA, a schematic model for the hDus2/tRNA complex 


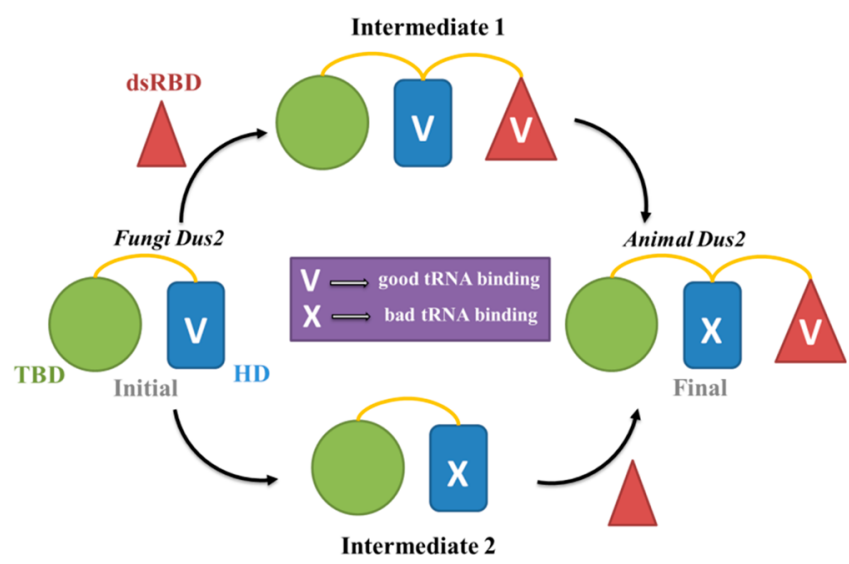

Figure 5. Speculative minimal evolutionary scenarios leading to the emergence of complex animal Dus2 modularity and a new tRNA binding mode among Dus enzymes. In this model, we propose that the common ancestor of both fungi and animal may have likely carried both the TBD and HD. The first pathway shows that the acquisition of a dsRBD occurred before the loss of positive charges in the $\mathrm{HD}$, leading to a putative intermediate 1 carrying too much affinity for tRNA. Then, this intermediate evolved to a more functional state by converting HD into a less electropositive domain. Conversely, the second pathway proposes that the loss of positive charges occurred first and then acquisition of a dsRBD, which restored a better functionality.

has been postulated. ${ }^{10}$ In the latter, the dsRBD together with the TBD and HD allow hDus2 to embrace almost the whole substrate. This new tRNA binding mode does not seem to present any obvious advantage in terms of stability for the complex since both Dus $2 p$ and hDus2 share a similar affinity for tRNA. Furthermore, we and other groups showed that DusA, ${ }^{19}$ Dus $2 p{ }^{22}$ and $h D u s 2^{18}$ are active only on mature tRNA bearing modifications and inactive on a tRNA transcript. This clearly suggests that the canonical Dus structure is endowed with a capacity to discriminate its substrate. Finally, having a dsRBD does not influence flavin reactivity and consequently the catalysis per se, comforting that its role is purely dedicated to tRNA binding. Thus, animal Dus2 represents a single class of modifying enzymes that has partially transferred its tRNA recognition function from a specialized domain, $\mathrm{HD}$, to a rather common dsRBD. This domain already present in $\mathrm{LUCA}^{29}$ has emerged as a promiscuous RNA binding module for several enzyme family-catalyzing unrelated chemistries. ${ }^{30}$ Perhaps, beyond the tRNA recognition, dsRBD acquisition may be needed for other unknown functions of Dus2, since in addition to being involved in dsRNA binding, dsRBDs are known to serve as a protein/protein interaction module in RNA metabolism enzymes, but this remains to be proven.

In the absence of clear alternative functions, what appears unambiguous at this stage is the important role played by the dsRBD in the tRNA recognition function of hDus2. Although there is currently no phylogenetic study addressing such a specific question, we would still like to speculate on possible scenarios that could explain how a novel tRNA binding mode emerged in animal Dus2. Even though several possibilities could have happened, we propose two rather simplistic mechanisms that seem plausible (Figure 5).

In the first scenario, the ancestral animal Dus 2 formed by $\mathrm{TBD}+\mathrm{HD}$, and closely related to its orthologous relatives in fungi, first acquires a dsRBD by gene fusion, a classical mechanism of protein modularity, leading to an intermediate protein $\mathbf{1}$ with a very high affinity for tRNA provided by the presence of two RNA binding domains. With this state being most likely not functional because of its excessive affinity that would affect the tRNA binding dynamic, intermediate 1 could have undergone mutations within the $\mathrm{HD}$ that decrease its electropositivity, generating animal Dus2. In the second scenario, HD first loses its electropositivity, weakening tRNA binding, and then functionality recovers after dsRBDs acquisition. In the latter case, the modularity could aim to rescue a compromised function. Although we have no evidence yet to support these hypotheses, we believe that ancestral enzyme reconstruction could clarify these fascinating issues and provide further insight into the evolutionary role of enzyme modularity.

\section{ASSOCIATED CONTENT}

\section{S Supporting Information}

The Supporting Information is available free of charge on the ACS Publications website at DOI: 10.1021/acs.biochem. 8 b00584.

Sequence alignment between Dus $2 \mathrm{p}$ and $\mathrm{hDus} 2^{\mathrm{TBD}+\mathrm{HD}}$, Dus2p homology models, electrostatic surfaces of Dus2 models and $\Delta(2-13) \mathrm{hDus} 2^{\mathrm{TBD}+\mathrm{HD}}$, sequence alignments of fungi Dus2 and animal Dus2 ${ }^{\mathrm{TBD}+\mathrm{HD}}$, UVvisible spectrum of $\mathrm{hDus} 2^{\mathrm{TBD}+\mathrm{HD}}$ and its mutants, gel filtration profiles of $\mathrm{hDus} 2^{\mathrm{TBD}+\mathrm{HD}}$ and its mutants, electrophoretic mobility shift assays of Dus2p, hDus $2^{\mathrm{TBD}+\mathrm{HD}}$, and mutants, tRNA binding isotherms, and STARANISO determination of best-fit ellipsoid to the anisotropic cut-off surface (PDF)

\section{AUTHOR INFORMATION}

\section{Corresponding Author}

*E-mail: djemel.hamdane@college-de-france.fr. Tel: +33-(0)144271278.

ORCID

Djemel Hamdane: 0000-0002-1737-8320

Funding

This work was supported by the Centre National de la Recherche Scientifique, Sorbonne Université as well as the "Initiative d'Excellence" program from the French State (Grant "DYNAMO", ANR-11-LABX-0011-01 and ANR-15-CE110004-01).

\section{Notes}

The authors declare no competing financial interest.

\section{ACKNOWLEDGMENTS}

We acknowledge SOLEIL for provision of the synchrotron radiation facilities (proposal ID 20150780), and we would like to thank Proxima 2 for assistance in using the beamline.

\section{REFERENCES}

(1) Hawkins, A. R., and Lamb, H. K. (1995) The molecular biology of multidomain proteins. Selected examples. Eur. J. Biochem. 232, $7-$ 18.

(2) Vogel, C., Bashton, M., Kerrison, N. D., Chothia, C., and Teichmann, S. A. (2004) Structure, function and evolution of multidomain proteins. Curr. Opin. Struct. Biol. 14, 208-216.

(3) Khosla, C., and Harbury, P. B. (2001) Modular enzymes. Nature 409, 247-252. 
(4) Chothia, C., Gough, J., Vogel, C., and Teichmann, S. A. (2003)

Evolution of the protein repertoire. Science 300, 1701-1703.

(5) Guo, M., Yang, X. L., and Schimmel, P. (2010) New functions of aminoacyl-tRNA synthetases beyond translation. Nat. Rev. Mol. Cell Biol. 11, 668-674.

(6) Perona, J. J., and Hadd, A. (2012) Structural diversity and protein engineering of the aminoacyl-tRNA synthetases. Biochemistry $51,8705-8729$.

(7) Nicholson, A. W. (2014) Ribonuclease III mechanisms of double-stranded RNA cleavage. Wiley Interdiscip Rev. RNA 5, 31-48.

(8) Court, D. L., Gan, J., Liang, Y. H., Shaw, G. X., Tropea, J. E., Costantino, N., Waugh, D. S., and Ji, X. (2013) RNase III: Genetics and function; structure and mechanism. Annu. Rev. Genet. 47, 405431.

(9) Grosjean, H. (2009) DNA and RNA Modification Enzymes: Structure, Mechanism, Function and Evolution, Landes Bioscience.

(10) Bou-Nader, C., Pecqueur, L., Bregeon, D., Kamah, A., Guerineau, V., Golinelli-Pimpaneau, B., Guimaraes, B. G., Fontecave, M., and Hamdane, D. (2015) An extended dsRBD is required for post-transcriptional modification in human tRNAs. Nucleic Acids Res. 43, 9446-9456.

(11) Dyubankova, N., Sochacka, E., Kraszewska, K., Nawrot, B., Herdewijn, P., and Lescrinier, E. (2015) Contribution of dihydrouridine in folding of the D-arm in tRNA. Org. Biomol. Chem. 13, 49604966.

(12) Jones, C. I., Spencer, A. C., Hsu, J. L., Spremulli, L. L., Martinis, S. A., DeRider, M., and Agris, P. F. (2006) A counterintuitive Mg2+dependent and modification-assisted functional folding of mitochondrial tRNAs. J. Mol. Biol. 362, 771-786.

(13) Alexandrov, A., Chernyakov, I., Gu, W., Hiley, S. L., Hughes, T. R., Grayhack, E. J., and Phizicky, E. M. (2006) Rapid tRNA decay can result from lack of nonessential modifications. Mol. Cell 21, 87-96.

(14) Lombard, M., and Hamdane, D. (2017) Flavin-dependent epitranscriptomic world. Arch. Biochem. Biophys. 632, 28-40.

(15) Kasprzak, J. M., Czerwoniec, A., and Bujnicki, J. M. (2012) Molecular evolution of dihydrouridine synthases. BMC Bioinf. 13, 153.

(16) Bishop, A. C., Xu, J., Johnson, R. C., Schimmel, P., and de Crecy-Lagard, V. (2002) Identification of the tRNA-dihydrouridine synthase family. J. Biol. Chem. 277, 25090-25095.

(17) Bou-Nader, C., Montemont, H., Guerineau, V., Jean-Jean, O., Bregeon, D., and Hamdane, D. (2018) Unveiling structural and functional divergences of bacterial tRNA dihydrouridine synthases: perspectives on the evolution scenario. Nucleic Acids Res. 46, 13861394.

(18) Byrne, R. T., Jenkins, H. T., Peters, D. T., Whelan, F., Stowell, J., Aziz, N., Kasatsky, P., Rodnina, M. V., Koonin, E. V., Konevega, A. L., and Antson, A. A. (2015) Major reorientation of tRNA substrates defines specificity of dihydrouridine synthases. Proc. Natl. Acad. Sci. U. S. A. 112, 6033-6037.

(19) Yu, F., Tanaka, Y., Yamashita, K., Suzuki, T., Nakamura, A., Hirano, N., Yao, M., and Tanaka, I. (2011) Molecular basis of dihydrouridine formation on tRNA. Proc. Natl. Acad. Sci. U. S. A. 108, 19593-19598.

(20) Kusuba, H., Yoshida, T., Iwasaki, E., Awai, T., Kazayama, A., Hirata, A., Tomikawa, C., Yamagami, R., and Hori, H. (2015) In vitro dihydrouridine formation by tRNA dihydrouridine synthase from Thermus thermophilus, an extreme-thermophilic eubacterium. J. Biochem. 158, 513-521.

(21) Xing, F., Hiley, S. L., Hughes, T. R., and Phizicky, E. M. (2004) The specificities of four yeast dihydrouridine synthases for cytoplasmic tRNAs. J. Biol. Chem. 279, 17850-17860.

(22) Rider, L. W., Ottosen, M. B., Gattis, S. G., and Palfey, B. A. (2009) Mechanism of dihydrouridine synthase 2 from yeast and the importance of modifications for efficient tRNA reduction. J. Biol. Chem. 284, 10324-10333.

(23) Betteridge, T., Liu, H., Gamper, H., Kirillov, S., Cooperman, B. S., and Hou, Y. M. (2007) Fluorescent labeling of tRNAs for dynamics experiments. RNA 13, 1594-1601.
(24) Chen, M., Yu, J., Tanaka, Y., Tanaka, M., Tanaka, I., and Yao, M. (2013) Structure of dihydrouridine synthase C (DusC) from Escherichia coli. Acta Crystallogr., Sect. F: Struct. Biol. Cryst. Commun. 69, 834-838.

(25) Whelan, F., Jenkins, H. T., Griffiths, S. C., Byrne, R. T., Dodson, E. J., and Antson, A. A. (2015) From bacterial to human dihydrouridine synthase: automated structure determination. Acta Crystallogr., Sect. D: Biol. Crystallogr. 71, 1564-1571.

(26) Kato, T., Daigo, Y., Hayama, S., Ishikawa, N., Yamabuki, T., Ito, T., Miyamoto, M., Kondo, S., and Nakamura, Y. (2005) A novel human tRNA-dihydrouridine synthase involved in pulmonary carcinogenesis. Cancer Res. 65, 5638-5646.

(27) Mittelstadt, M., Frump, A., Khuu, T., Fowlkes, V., Handy, I., Patel, C. V., and Patel, R. C. (2007) Interaction of human tRNAdihydrouridine synthase- 2 with interferon-induced protein kinase PKR. Nucleic Acids Res. 36, 998-1008.

(28) Adams, P. D., Afonine, P. V., Bunkoczi, G., Chen, V. B., Davis, I. W., Echols, N., Headd, J. J., Hung, L. W., Kapral, G. J., GrosseKunstleve, R. W., McCoy, A. J., Moriarty, N. W., Oeffner, R., Read, R. J., Richardson, D. C., Richardson, J. S., Terwilliger, T. C., and Zwart, P. H. (2010) PHENIX: a comprehensive Python-based system for macromolecular structure solution. Acta Crystallogr., Sect. D: Biol. Crystallogr. 66, 213-221.

(29) Vonrhein, C., Flensburg, C., Keller, P., Sharff, A., Smart, O., Paciorek, W., Womack, T., and Bricogne, G. (2011) Data processing and analysis with the autoPROC toolbox. Acta Crystallogr., Sect. D: Biol. Crystallogr. 67, 293-302.

(30) Smart, O. S., Womack, T. O., Flensburg, C., Keller, P., Paciorek, W., Sharff, A., Vonrhein, C., and Bricogne, G. (2012) Exploiting structure similarity in refinement: automated NCS and targetstructure restraints in BUSTER. Acta Crystallogr., Sect. D: Biol. Crystallogr. 68, 368-380.

(31) Emsley, P., Lohkamp, B., Scott, W. G., and Cowtan, K. (2010) Features and development of Coot. Acta Crystallogr., Sect. D: Biol. Crystallogr. 66, 486-501.

(32) Yang, J., Yan, R., Roy, A., Xu, D., Poisson, J., and Zhang, Y. (2015) The I-TASSER Suite: protein structure and function prediction. Nat. Methods 12, 7-8.

(33) Waterhouse, A., Bertoni, M., Bienert, S., Studer, G., Tauriello, G., Gumienny, R., Heer, F. T., de Beer, T. A. P., Rempfer, C., Bordoli, L., Lepore, R., and Schwede, T. (2018) SWISS-MODEL: homology modelling of protein structures and complexes. Nucleic Acids Res. 46, W296-W303.

(34) Kelley, L. A., Mezulis, S., Yates, C. M., Wass, M. N., and Sternberg, M. J. (2015) The Phyre2 web portal for protein modeling, prediction and analysis. Nat. Protoc. 10, 845-858.

(35) Kim, D. E., Chivian, D., and Baker, D. (2004) Protein structure prediction and analysis using the Robetta server. Nucleic Acids Res. 32, W526-531.

(36) Uziela, K., Menendez Hurtado, D., Shu, N., Wallner, B., and Elofsson, A. (2017) ProQ3D: improved model quality assessments using deep learning. Bioinformatics 33, 1578-1580.

(37) Baker, N. A., Sept, D., Joseph, S., Holst, M. J., and McCammon, J. A. (2001) Electrostatics of nanosystems: application to microtubules and the ribosome. Proc. Natl. Acad. Sci. U. S. A. 98, 1003710041.

(38) Dolinsky, T. J., Czodrowski, P., Li, H., Nielsen, J. E., Jensen, J. H., Klebe, G., and Baker, N. A. (2007) PDB2PQR: expanding and upgrading automated preparation of biomolecular structures for molecular simulations. Nucleic Acids Res. 35, W522-525. 